\title{
Modelos de provisión y formación dual. Una tarea pendiente para el caso de la enseñanza media técnico-profesional en Chile
}

\author{
Dual Provision and Training Models. A Pending Task for the Case of \\ Professional Technical Secondary Education in Chile
}

\author{
Christian Loyola-Bustos ${ }^{1}$
}

\begin{abstract}
RESUMEN
El objetivo del presente trabajo es conocer y analizar los modelos de provisión que se despliegan en la enseñanza media técnico-profesional (EMTP), relacionando estos modelos con la formación dual y teniendo como referencia la experiencia alemana. La metodología consistirá en la revisión de bibliografía especializada. La enseñanza técnico profesional es fundamental para el desarrollo e inserción laboral de los y las jóvenes, es por esto que se deben mejorar los modelos de provisión en Chile, con el fin de que la EMTP sea eficiente y efectiva en su preparación y estructura. El artículo busca contribuir a la mejora educativa, recalcando la importancia de establecer conexiones, redes y vínculos entre empresas y dependencias educativas, propiciando un aprendizaje dual, con el fin de que la formación de los estudiantes sea de calidad e impacte de manera positiva el mercado laboral.
\end{abstract}

Palabras claves: enseñanza técnico profesional; modelos de provisión; educación y trabajo; formación dual; proyecciones laborales.

\begin{abstract}
The objective of this work is to know and analyze the provision models that are deployed in professional technical secondary education (EMPT in Spanish),, relating these models to dual training, taking the German experience as a reference. The methodology will consist of the review of specialized bibliography. Professional technical education is essential for the development and job placement of young people, which is why the provision models in Chile must be improved, in order for the EMTP to be efficient and effective in its preparation and structure. The article seeks to contribute to educational improvement, stressing the importance of establishing connections, networks and ties between companies and educational agencies, promoting dual learning, so that the education offered is of good quality impacting positively the labor market.
\end{abstract}

Keywords: Professional technical education; models of provision; education and work; dual training; labor projections.

1 Pontificia Universidad Católica de Valparaíso, Valparaíso, Chile; magíster en Política Educativa; loyola.cristian.d@gmail.com. 
MODELOS DE PROVISIÓN Y FORMACIÓN DUAL. UNA TAREA PENDIENTE PARA EL CASO DE LA ENSEÑANZA MEDIA TÉCNICO-PROFESIONAL EN CHILE / LOYOLA-BUSTOS

\section{Introducción}

De acuerdo con Leandro Sepúlveda, "un rasgo característico de las principales transformaciones sociales ocurridas en Chile y el resto de América Latina en los últimos años dice relación con la expansión de los sistemas educativos y el incremento de la escolaridad de la población" (Sepúlveda, 2008, p.11; Carnoy, 1999; Hargreaves y Shirley, 2012). En esta misma línea, al referirse al caso chileno, el autor afirma que

la cobertura de la educación básica ha alcanzado niveles de universalización y, en la educación secundaria, los indicadores reflejan cambios significativos en las últimas dos décadas, dando cuenta de un hecho relevante: este sistema educacional ha dejado de ser un espacio formativo al que accedía solo un segmento menor de la población e incluso, desde un tiempo a esta parte, se hace evidente que la enseñanza media es una etapa imprescindible, aunque no suficiente, para una exitosa integración social e inicio de la vida laboral de las personas (Sepúlveda, 2008, p.11).

Ahora bien, cabe tener presente que la masificación del nivel de enseñanza media está asociada, entre otras razones, "al incremento proporcional de la matrícula de la educación media técnico profesional, lo que significó el aumento de la proporción de representatividad desde el 34,4\% en el año 1990, al 45,1\% en el año 1998" (Mineduc, 2007, citado en Sepúlveda, 2008, p.12).

El presente artículo se refiere al sistema de la Educación Media Técnico Profesional (en adelante EMTP) chileno y tiene como eje central su modelo de provisión, el acompañamiento que se hace a los/as estudiantes y el rol que tiene la formación dual en la enseñanza técnico profesional (en adelante ETP), entendiendo que esta puede ser una alternativa para las demandas del sector productivo del país (Paredes y Sevilla, 2015).

El objetivo del presente trabajo es conocer y analizar los modelos de provisión que se dan en la EMTP, resaltando la importancia de la formación dual alemana y sus posibles proyecciones en Chile. La metodología consistirá en la revisión de bibliografía especializada.

En función de la política pública educativa, resulta imprescindible enriquecer esfuerzos asociados a establecer redes de articulación vinculadas a la formación dual, ya que los 
REVISTA SABERES EDUCATIVOS, Nº 5, JULIO-DICIEMBRE 2020

resultados evidencian una mejora en la calidad de la ETP a partir de esta articulación del sistema (Sevilla, Farías y Weintraub, 2014). Consideramos que la ETP es fundamental para el desarrollo económico e inserción laboral de los jóvenes, es por esto que se deben mejorar los modelos de provisión en Chile, con el fin de que la EMTP sea eficiente y efectiva en su preparación y estructura. De ahí que el presente estudio busca contribuir a la mejora educativa, recalcando la importancia de establecer conexiones, redes, vínculos con empresas, con el fin de que la formación de los estudiantes de la EMTP sea de calidad e impacte de manera positiva el mercado laboral.

\section{La EMTP: diagnóstico y articulación}

Existe un cierto grado de dificultad para definir lo que es la ETP, debido a que las fronteras en el ámbito educativo no son por lo general precisas (Sevilla, 2017). De acuerdo con la OCDE (2010a), una definición empleada con frecuencia es la que señala que la educación técnico profesional combina "el aprendizaje teórico y práctico para una ocupación o campo ocupacional específico, distinguiendo entre ETP inicial ${ }^{2}$ y continua” (Sevilla, 2017, p.11).

De acuerdo con Moodie (2008), en América Latina los modelos institucionales que provisionan la ETP son determinados por los mecanismos de mercado que regulan la asignación de recursos en cada país. Según Sevilla (2017), en las

economías de mercado liberales, donde el mercado asume el rol de seleccionar y vincular la demanda y la oferta laboral, es esperable que la separación entre la educación técnica y académica sea tenue y tardía. En cambio, en economías coordinadas en las que el Estado y las cámaras empresariales son las responsables de definir la provisión de formación y capacitación laboral alineándola con el empleo, los estudiantes son separados tempranamente e impera un modelo segmentado de provisión de las vías académica y técnico profesional. (p.13)

\footnotetext{
2 "La primera incluye los programas formales de nivel secundario y superior, diseñados para ser impartidos a los jóvenes al inicio de sus trayectorias profesionales y previo al ingreso al mercado del trabajo" (Sevilla, 2017, p. 11).
} 
MODELOS DE PROVISIÓN Y FORMACIÓN DUAL. UNA TAREA PENDIENTE PARA EL CASO DE LA ENSEÑANZA MEDIA TÉCNICO-PROFESIONAL EN CHILE / LOYOLA-BUSTOS

En Chile la EMTP se ha caracterizado por tener un modelo de provisión segmentado, es decir, se dividen "los planes de estudio de la secundaria en académicos y técnicos, los que se imparten de manera paralela, ya sea en una misma unidad educacional o en establecimientos separados" (Sevilla, 2017, p.13). Según la misma autora, "el nexo entre ambas ofertas formativas es limitado, así como también los vínculos con la ETP superior. Lo que se intenta es estrechar la relación con el sector productivo para asegurar la pertinencia de esta educación con sus requerimientos" (Sevilla, 2017, p.13).

De acuerdo a la Clasificación Internacional Normalizada de Educación (CINE), en la región latinoamericana, las modalidades más comunes que imponen un sello a la oferta de ETP pueden ser de tres tipos: programas de ETP en la secundaria baja (CINE 1), programas de ETP profesionalizantes en la secundaria baja y alta (CINE 2 y 3), y programas optativos de profundización de ETP en la secundaria alta (CINE 3) ${ }^{3}$.

En relación a la modalidad dominante de la EMTP, la que impera es la que tiene base "en la escuela propedéutica o híbrida, en el sentido de que combina contenidos de formación general con cursos técnicos profesionales asociados a campos ocupacionales específicos y que habilita tanto para la inserción laboral como para la continuidad de estudios" (Sevilla, 2017, p.21; Jacinto, 2013). Es así como, en el caso chileno, la EMTP está legalmente “abierta a la educación superior y promete a los jóvenes y a las familias que la eligen una formación integral en el ámbito de una especialidad que facilite su inserción laboral, así como también la posibilidad de continuación de estudios" (Sevilla et al., 2014, p.84; Larrañaga, Cabezas y Dussaillant, 2013; Mineduc, 2011).

Conforme a un estudio realizado en Chile por las autoras Camila Arrollo y Francisca Pacheco (2018):

En 2016, 2.874 establecimientos impartían educación media. Un 33\% (948) impartía EMTP, ya sea en modalidad especializada (solo EMTP) o polivalente (es decir,

\footnotetext{
${ }^{3}$ De acuerdo con Paola Sevilla, Chile se encontraría en esta categoría, en donde "los estudiantes graduados de los programas de ETP de carácter propedéutico pueden, de manera opcional, cursar un año adicional de estudios secundarios. Ello les permite especializarse en un campo ocupacional determinado y adquirir otra certificación, pero no necesariamente significa una progresión en sus estudios formales” (Sevilla, 2017, p.23).
} 
REVISTA SABERES EDUCATIVOS, Nº 5, JULIO-DICIEMBRE 2020

establecimientos que imparten ambas modalidades, científico humanista $(\mathrm{CH})$ y técnico profesional (TP)), y en ellos se concentraba el 39\% de la matrícula de enseñanza media (158.537 alumnos). (p.5)

Durante los años 2004-2016, las dependencias educativas que entregan una formación técnico-profesional "[han] aumentado en un $26 \%$. Sin embargo, se observa un estancamiento en la oferta de establecimientos a contar del año 2012 [Figura 1], a diferencia de los establecimientos que imparten educación media científico-humanista". (Arrollo y Pacheco, 2018, p.5).

\section{Figura 1}

Evolución del número de establecimientos con educación media científico humanista (CH), técnico profesional (TP) y polivalentes.

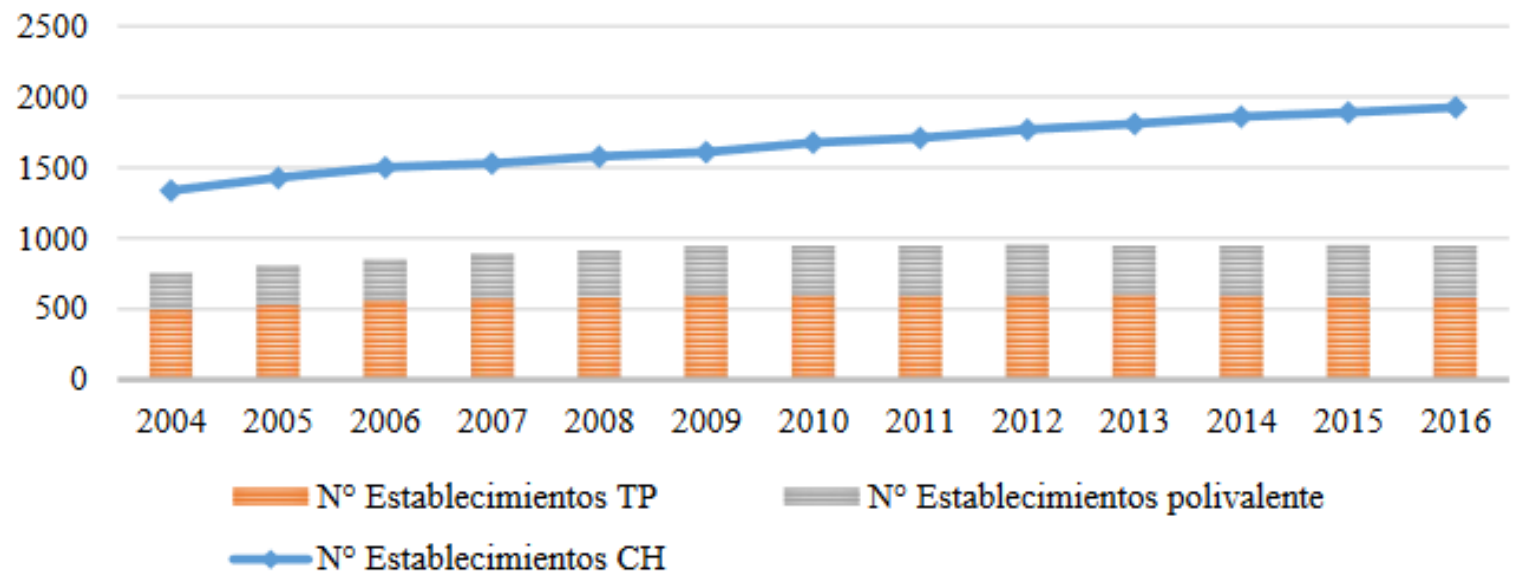

Nota. Tomado de Evolución del número de establecimientos CH-TP y polivalentes (p.6), por C. Arrollo y F. Pacheco, 2018, Comisión Nacional de Productividad, Santiago de Chile.

Según las autoras, en el año 2016, del total de "establecimientos que impartían educación media técnico profesional, el 50\% correspondía a liceos técnicos municipales, el $43 \%$ a establecimientos particulares subvencionados y el 7\% a Corporaciones de Administración Delegada (70 establecimientos)" (Arrollo y Pacheco, 2018, p.6), es decir, a “establecimientos 
MODELOS DE PROVISIÓN Y FORMACIÓN DUAL. UNA TAREA PENDIENTE PARA EL CASO DE LA ENSEÑANZA MEDIA TÉCNICO-PROFESIONAL EN CHILE / LOYOLA-BUSTOS

educacionales de propiedad del Estado que son financiados a través de convenios de administración suscritos por entidades de derecho privado, vinculadas al mundo empresarial e industrial" (Sepúlveda, 2008, p.21).

En la Tabla 1 se puede observar que del "total de establecimientos municipales que imparten educación a jóvenes de tercero y cuarto medio, el $60 \%$ ofrece la modalidad técnico profesional como alternativa de enseñanza, en contraste con el $25 \%$ de los establecimientos particulares subvencionados" (Arrollo y Pacheco, 2018, p.6).

\section{Tabla 1}

Establecimientos de educación media, según dependencia y modalidad (2016)

\begin{tabular}{rccccc}
\hline $\begin{array}{c}\text { Tipo de } \\
\text { establecimiento }\end{array}$ & Municipal & $\begin{array}{c}\text { Particular } \\
\text { subvencionado }\end{array}$ & $\begin{array}{c}\text { Particular } \\
\text { pagado }\end{array}$ & $\begin{array}{c}\text { Administración } \\
\text { delegada }\end{array}$ & Total \\
\hline Científico humanista & 323 & 1.215 & 388 & 0 & 1.926 \\
Técnico profesional & 245 & 265 & 0 & 70 & 580 \\
Polivalente & 226 & 142 & 0 & 0 & 368 \\
Total & 794 & 1.622 & 388 & 70 & 2.874 \\
\hline
\end{tabular}

Nota. Adaptado de Establecimientos de educación media, según dependencia y modalidad, 2016 (p.6), por C. Arrollo y F. Pacheco, 2018, Comisión Nacional de Productividad, Santiago de Chile.

La EMTP “involucra una participación significativa de jóvenes estudiantes y, por ende, tiene un rol fundamental en la formación de la futura fuerza laboral chilena" (Arias, Farías, González-Velosa, Hunneus y Rucci et. al., 2015, p.3). En nuestro país, el 33\% de los/as estudiantes de tercero y cuarto medio eligen la modalidad de EMTP como opción (Arias et al., 2015). Al respecto, los autores exponen:

Esta proporción es el doble de la de otros países de la región y cercana al promedio de países de la OCDE. Además, un 38\% de la matrícula de primer año de educación superior corresponde a programas de la modalidad de educación superior técnico 
REVISTA SABERES EDUCATIVOS, № 5, JULIO-DICIEMBRE 2020

profesional, en un sistema en el cual la tasa neta de participación en educación superior es del 70.7\%. (Arias et al., 2015, p.3)

Lo anterior se condice con la evaluación de la EMTP por parte de la opinión pública en los sectores socioeconómicos más bajos, con una muy buena percepción (Arancibia, 1994; López, Elizalde y Rolando, 2015; Paredes y Sevilla, 2015).

Como se señaló anteriormente, los modelos de provisión que emergen en la ETP son básicamente dos: uno especializado y otro polivalente. "Para el año 2016, de los 2.874 establecimientos educacionales que impartían educación media, 368 eran polivalentes. Cabe destacar que la participación de este tipo de establecimientos en la provisión de educación media ha aumentado sustantivamente en los últimos 10 años" (Arrollo y Pacheco, 2018, p.8), tomando como referencia los años 2006-2016, como muestra la Figura 2.

\section{Figura 2}

Establecimientos de educación media según dependencia administrativa (2004 - 2016)

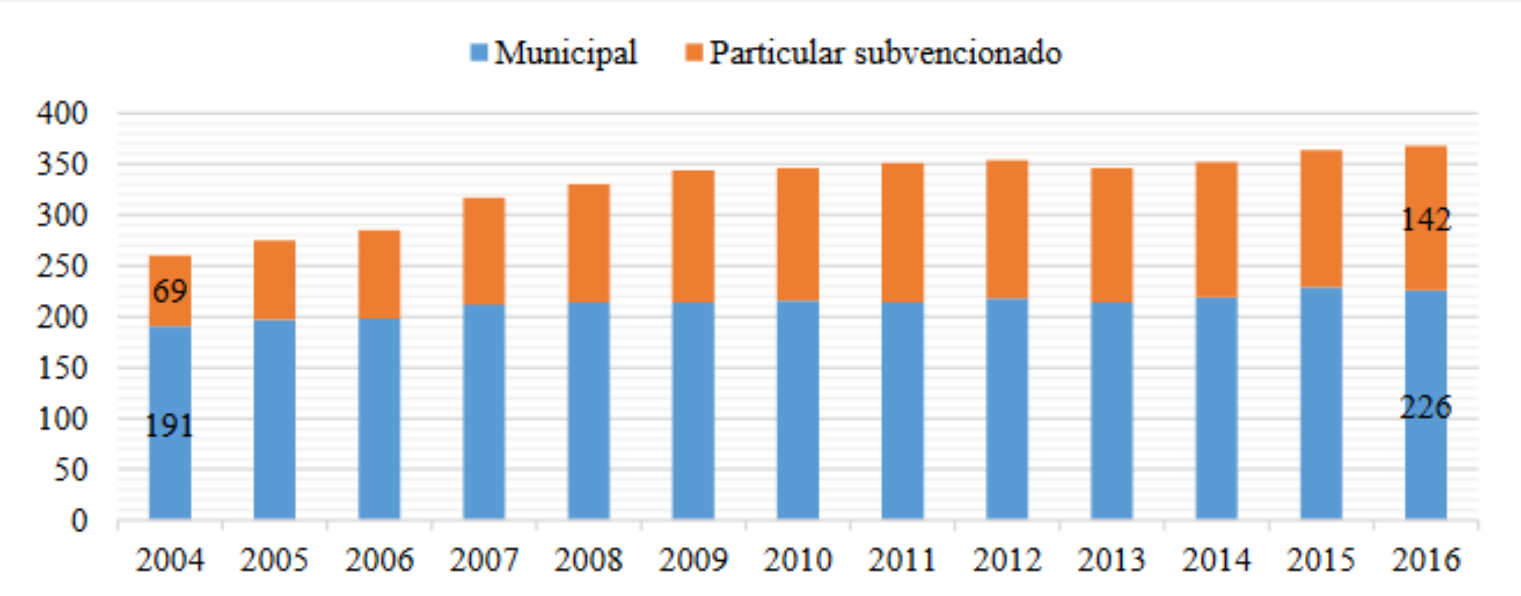

Nota. Tomado de Número de establecimientos polivalentes de educación media, según dependencia administrativa, 2004-2016 (p.9), por C. Arrollo y F. Pacheco, 2018, Comisión Nacional de Productividad, Santiago de Chile. 
MODELOS DE PROVISIÓN Y FORMACIÓN DUAL. UNA TAREA PENDIENTE PARA EL CASO DE LA ENSEÑANZA MEDIA TÉCNICO-PROFESIONAL EN CHILE / LOYOLA-BUSTOS

Sepúlveda (2008) afirma que concentración de la matrícula se da "en las especialidades de administración (cerca del $40 \%$ de la matrícula total), seguida de electricidad y metalmecánica, con un poco más del 27\% de la matrícula entre ambos sectores” (Sepúlveda, 2008, p. 22). Asimismo,

es destacable la incidencia del sector servicios en la actual estructura de la matrícula EMTP. Si se suman los sectores de administración, programas sociales, alimentación, hotelería y turismo, el porcentaje se empina al 57\% del total, configurando un cuadro muy distinto al registrado en décadas pasadas, donde existía una fuerte incidencia de las carreras ligadas al sector industrial. (Sepúlveda, 2008, p.22)

Con relación a los y las docentes, en general, los sistemas de ETP a nivel internacional se han visto envueltos en el debate sobre el reclutamiento y selección de los/as docentes que impartirán esos conocimientos y habilidades esenciales. La experiencia de los/as docentes ha sido el verdadero telón de Aquiles del sistema de ETP y en algunos casos esto no ha sido tomado en cuenta (Arancibia et al., 2008).

De acuerdo al trabajo desarrollado por el Consejo Asesor Presidencial para la Calidad de la Educación (2006), citado por Sepúlveda (2008),

la formación general que se imparte en $1^{\circ}$ y $2^{\circ}$ medio en establecimientos EMTP no es equivalente a la que se ofrece en establecimientos EMCH (...). De igual manera, se constata que muchos establecimientos EMTP exigen que los estudiantes que ingresan en $1^{\circ}$ medio elijan anticipadamente su especialidad técnica, contrario al requisito que esto sea al término del $2^{\circ}$ año medio. Esta situación se explicaría por la necesidad que tienen los establecimientos EMTP de asegurar una demanda mínima para las distintas opciones de especialización que imparten (...). Finalmente, dicho informe advierte que la mayoría de los docentes que trabajan en las especialidades EMTP no poseen formación pedagógica, indicándose que cerca del $20 \%$ de estos profesores corresponde a estudiantes egresados de los mismos establecimientos (Sepúlveda, 2008, p.23). 


\section{Educación y trabajo: trayectorias laborales en la educación media técnico-profesional y la formación dual}

Un elemento central de la EMTP es la transición que hacen los estudiantes desde la escuela al trabajo. De ahí la importancia y valoración que se le da a la obtención de un título en la educación media con la posibilidad de inserción laboral temprana (Montecinos, 2017). De acuerdo a los estudios realizados por Sepúlveda y Valdebenito (2014a; 2014b), "la gran mayoría de los jóvenes chilenos, independiente del establecimiento donde estudiaron o el nivel-socioeconómico al que pertenecen, aspiran a cursar estudios superiores una vez finalizada su enseñanza secundaria” (Sepúlveda y Valdebenito, 2014a, p.259).

La preparación para el trabajo y el desarrollo de competencias laborales, para los autores, corresponde a

una dimensión altamente valorada por los estudiantes de la EMTP, reforzando la importancia de contar con un sistema de educación diferenciado a nivel de la educación secundaria en nuestro país. Con todo, la complejidad y heterogeneidad de este sistema formativo, particularmente en lo referido a los diversos sectores productivos que concurren en su oferta curricular, permiten observar diferencias importantes en la orientación vocacional y proyección de futuro de los estudiantes. (Sepúlveda y Valdebenito, 2014b, p. 615)

La EMTP a nivel internacional tiene una fuerte base en "la institución escolar y el aprendizaje en los lugares de trabajo está limitado por pasantías o prácticas profesionales, las cuales tienen un alcance temporal parcial y, en algunos casos, no son requisito de graduación” (Sevilla, 2017, p.39). Para el caso chileno, estas pasantías se desarrollan luego de ser promovidos de $2^{\circ}$ medio, entrando a una etapa seudoelectiva. No obstante, el paso por la empresa (inserción) se da solo al momento de la realización de la práctica profesional.

A pesar de esto, en nuestro sistema escolar hay excepciones que son significativas y apuntan al aprendizaje dual. La formación dual consiste en un tipo de modalidad que se da en la EMTP, basada en la alternancia curricular. Por una parte, la formación de conocimientos y habilidades curriculares desarrolladas en la dependencia educativa y, por otro lado, de 
MODELOS DE PROVISIÓN Y FORMACIÓN DUAL. UNA TAREA PENDIENTE PARA EL CASO DE LA ENSEÑANZA MEDIA TÉCNICO-PROFESIONAL EN CHILE / LOYOLA-BUSTOS

manera coordinada y en conjunto, la inserción de los/as estudiantes en el mundo productivo (la empresa), adquiriendo allí aquellas habilidades específicas propias de la disciplina. A raíz de lo anterior, se afirma que los/as estudiantes

pueden asistir en regímenes de tres días al liceo y dos días a la empresa, o bien, una semana al liceo y una semana a la empresa. Las empresas reciben a uno o más “aprendices" durante dos años seguidos, a partir de $3^{\circ}$ medio, respetando su condición de alumnos del liceo. El modelo se implementó en Chile en 1992 como una iniciativa de la Sociedad Alemana de Cooperación Técnica (GTZ), para fortalecer la EMTP en términos de calidad y vinculación con el mundo productivo. Una evaluación a la implementación de la formación dual en Chile realizada en el año 2000, cuando 78 liceos TP y 2.273 alumnos participaban de dicho modelo, dio cuenta de importantes beneficios asociados a esta iniciativa en relación a mejores tasas de inserción laboral de sus egresados. (Mineduc, 2011, p.19)

Respecto a esta modalidad, Dieter (2013b) profundiza aún más afirmando que [el] concepto de dualidad puede referirse a los lugares de aprendizaje, pero también a procesos didácticos entrelazados entre sí. En el primer caso, se hace referencia a la alternancia de aprendizaje en la empresa y la escuela (quedando de lado la importancia de otros lugares de aprendizaje). En el segundo caso se alude a la unión de teoría y práctica, de sistemática y casuística en el sentido de un "principio dual". De esta manera, el principio dual se puede implantar independientemente de la modalidad de formación. (p. 17)

La incidencia de la formación dual en nuestro sistema educativo es baja, por lo que se necesita revisar con claridad el mundo productivo, con el fin de especificar las posibilidades que tiene esa modalidad educativa. No cabe duda que los resultados esperados serán enriquecedores para los/as estudiantes, fortaleciendo el desarrollo integral (Lauglo, 2006). Es vital, desde esta perspectiva, ampliar y mejorar la vinculación de la EMTP con el desarrollo productivo, estableciendo ofertas que sean razonables y seduzcan a sus futuros/as estudiantes (Urzúa, 2012). En relación a este vínculo Villar (2013) señala: 
REVISTA SABERES EDUCATIVOS, Nº 5, JULIO-DICIEMBRE 2020

El vínculo usual puede tener dos etapas: la primera consiste en el vínculo colegioempresa, mientras que la segunda se asocia al vínculo universidad-empresa. Ambas integraciones buscan aminorar costos, a raíz del diálogo entre la esfera educativa (lo que se enseña) y la productiva (lo que se necesita). Si bien la segunda etapa puede ser la más productiva en términos de innovación, la primera puede sentar precedentes de una relación entre la empresa y el estudiante que orienten en un nivel máximo su praxis educativa, ya sea para su continuidad o nueva búsqueda de empleo. (p. 39)

En la misma lógica, "las empresas podrían aquí jugar un rol preponderante, por ejemplo, promoviendo por rubros soluciones a problemas cotidianos en los procesos productivos de las distintas áreas" (Villar, 2013, p. 39). Este incentivo tendría un mayor impacto tanto en el aprendizaje como en sus resultados. En este sentido, el modelo alemán dual cumple plenamente con los requisitos ${ }^{4}$ (Dieter, 2013a).

América Latina no se ha quedado atrás en búsquedas de implementación del modelo dual alemán, no obstante, de los países que contaron con asesoría, "solo Chile y Costa Rica lograron institucionalizar sus experiencias piloto y mantener en el tiempo esta formación como parte de su oferta de ETP regular" (Sevilla, 2017, p.40).

\section{La formación dual alemana en la educación técnico-profesional: hacia una EMTP de calidad en Chile}

No es de extrañar que durante los últimos años la EMTP haya estado en la palestra del debate público. "Los análisis son coincidentes en diagnosticar que este sistema formativo, que atiende a casi la mitad de los estudiantes de $3^{\circ}$ y $4^{\circ}$ medio, presenta serias debilidades" (Sepúlveda, Sevilla y Farías, 2014, p.1), lo que abre la posibilidad de creación de proyectos educativos y laborales que sean acordes a las necesidades propias de nuestra sociedad. Es por esto que se hace necesario reflexionar sobre la formación dual en la EMTP.

\footnotetext{
${ }^{4}$ Resulta fundamental que las empresas de distintas áreas busquen este tipo de establecimientos con mano de obra calificada y con una articulación efectiva entre los distintos niveles educativos.
} 
MODELOS DE PROVISIÓN Y FORMACIÓN DUAL. UNA TAREA PENDIENTE PARA EL CASO DE LA ENSEÑANZA MEDIA TÉCNICO-PROFESIONAL EN CHILE / LOYOLA-BUSTOS

En el caso de Alemania, "la ETP goza de un profundo respeto" (Sevilla, 2017, p.16; Hoeckel y Schwartz, 2010). En relación a las características principales, Sevilla (2017) afirma:

A) Una de las principales características del sistema educativo alemán es la separación temprana de sus estudiantes, los que siguen itinerarios diferenciados después de la educación primaria. El sistema se basa en la idea de que el alumno puede desarrollarse en el tipo de escuela acorde con sus necesidades y capacidades.

B) Dependiendo del tipo de escuela de origen, en la secundaria superior los estudiantes pueden seguir la vía académica impartida en el Gymnasium de ciclo superior o la vía profesional que se ofrece en el Berufsschule, tanto en la modalidad de jornada completa en la escuela o en el régimen de alternancia en la empresa.

C) El $60 \%$ de los estudiantes opta por la ruta profesional, prevaleciendo la modalidad de alternancia (dualidad) como la principal opción para los jóvenes.

D) En el régimen dual los estudiantes eligen entre 350 carreras de ETP, homologadas y certificadas en el sistema, que incluyen la combinación de formación teórica con la formación práctica directa en puestos de trabajo (Sevilla, 2017, p.16; CEDEFOP, 2012).

Según Rindfleish y Meannig-Fortmann (2015), "la formación dual prepara a los egresados de los colegios para la futura vida laboral" (p.9). En Alemania, los/as estudiantes deben procurar de manera autónoma una plaza en alguna empresa, buscando aquellos oficios que mejor satisfagan su interés. En este proceso

se debe escoger el oficio que se quiere aprender: en total existen aproximadamente 330 oficios diferentes (...). Una vez que se ha escogido el oficio, los estudiantes deben postularse directamente a las empresas para conseguir una plaza de aprendizaje. (...) los alumnos deben realizar una postulación por escrito, de la misma manera que si se tratara de un trabajo. (Rindfleish y Meannig-Fortmann, 2015, p.12) 
REVISTA SABERES EDUCATIVOS, Nº 5, JULIO-DICIEMBRE 2020

De esta manera, la adquisición de la experiencia de los/as futuros/as profesionales resulta fundamental. La formación dual va más allá de este ir y venir ya que fortalece de forma íntegra aquellas habilidades que son aprendidas, comprendidas y ejecutadas en el mundo del trabajo (Rindfleish y Meannig-Fortmann, 2015).

La modalidad de formación dual "ofrece a los jóvenes múltiples opciones de desarrollo posterior, en la medida que está estrechamente vinculado con el sistema educacional global" (Rindfleish y Meannig-Fortmann, 2015, p. 20). Con el aprendizaje dual, se afianza en los/as estudiantes el deseo de proseguir estudios universitarios y otros.

De acuerdo con un informe de la OCDE, las principales fortalezas de la educación dual en Alemania radican en altos niveles de participación del empresariado: "El sistema de la Educación y Formación Profesional en su conjunto cuenta con una cantidad adecuada de recursos que combinan la financiación pública y privada" (OCDE, 2010b, p.1). Desde una mirada gubernamental, la política educativa ha mantenido un fuerte respaldo económico en la oferta de programas de Educación y Formación Profesional, incluso en tiempos de crisis financiera.

\section{Proyecciones y reflexiones finales}

En primer lugar, es posible sostener que la formación dual debe articularse e implementarse en la EMTP chilena. Es imprescindible "atender a las diversas proyecciones de los jóvenes que acceden a esta opción formativa en busca de un espacio que reconozca sus intereses y aspiraciones vocacionales" (Sevilla et al., 2014, p.2). A partir de la evidencia, la implementación de la formación dual permite repensar la EMTP, estableciendo mejoras a las políticas públicas vinculadas a la formación técnico profesional.

En este sentido, es relevante declarar la EMTP fundamental para el desarrollo productivo. Desde esta perspectiva, el rol que tiene la política pública es importantísimo, entregando e invirtiendo recursos en estrategias concretas para avanzar en ello. Los/as estudiantes que buscan "acceder al mercado laboral inmediatamente después de concluir sus estudios secundarios, necesitan contar con una opción que los acerque de manera más directa y 
MODELOS DE PROVISIÓN Y FORMACIÓN DUAL. UNA TAREA PENDIENTE PARA EL CASO DE LA ENSEÑANZA MEDIA TÉCNICO-PROFESIONAL EN CHILE / LOYOLA-BUSTOS

sistemática a sus futuros puestos de trabajo" (Sevilla et al., 2014, p.2). En el caso chileno, hay serias deficiencias respecto a los espacios formativos de la EMTP. Los intereses de los/as estudiantes quedan sujetos y predispuestos a una limitada oferta de programas con escasas prácticas en centros o empresas vinculadas a esa oferta.

En segundo lugar, se sugiere asumir la formación dual como una estrategia de política pública. Al mismo tiempo, se recomienda "reconceptualizar la formación dual como un esquema flexible que se ensamble y adecue a los requerimientos de los sectores productivos" (Sevilla et al., 2014, p.3).

En tercer lugar, se deben intervenir escenarios posibles para una futura implementación de planes pilotos o transición respecto al aprendizaje dual en la EMTP chilena. Si bien se han desarrollado programas de dualidad en EMTP, estos aún son puntuales, no abarcando a la totalidad de los establecimientos educacionales.

En cuarto lugar, se deben generar condiciones para una formación sólida respecto a los/as jóvenes que acceden a esta modalidad de enseñanza, evidenciando la heterogeneidad en ofertas en estrecha relación con los mercados laborales (Sevilla, Farías y Sepúlveda, 2013; Messina, Weinberg e Irigoin, 1996).

En quinto lugar, se debe evitar la deserción y asegurar suficientes puestos de aprendizaje durante recesiones y aumentar la calidad de los/as docentes y formadores/as (Van Breugel, 2014). Sin duda, respecto a la formación docente, todavía quedan algunos vacíos importantes que terminan impactando en la calidad de los aprendizajes.

En sexto lugar, los tiempos actuales reclaman adaptarse con velocidad a los cambios en el sector productivo y a la introducción de nuevas tecnologías, y actualizar la propuesta educativa y curricular (Arias, Bassi y Covacevich, 2013).

Finalmente, la formación dual en EMTP debe convertirse en una prioridad y es perfectamente factible, siempre y cuando se puedan mejorar los puntos antes mencionados. Esa factibilidad depende principalmente de una voluntad, tanto política como social, de fortalecer cada día más este tipo de modalidad educativa, entendiendo que los modelos de provisión y las trayectorias laborales son una tarea pendiente en nuestro sistema educativo. 


\section{Referencias}

Arancibia, V. (1994). La Educación en Chile. Percepciones de la opinión pública y de expertos. Estudios Públicos, (54), 125-151. Recuperado de https://www.cepchile.cl/cep/site/artic/20160303/asocfile/20160303183853/rev54_ar ancibia.pdf

Arancibia, M. et al. (2008). Necesidades de formación permanente de docentes técnicos. Revista Estudios Pedagógicos, (34), 7-26. Recuperado de http://revistas.uach.cl/pdf/estped/v34n1/art01.pdf

Arias, E., Bassi, M. y Covacevich, C. (2013). Una buena educación técnica empieza por... BID, (21). Recuperado de http://idbdocs.iadb.org/wsdocs/getdocument.aspx?docnum=37951456

Arias, E., Farías, M., González-Velosa, C., Hunneus, C. y Rucci, G. (2015). Educación Técnico Profesional en Chile. Santiago, Chile: Banco Interamericano de Desarrollo (BID). Recuperado de https://publications.iadb.org/publications/spanish/document/Educaci\%C3\%B3nt\%C3\%A9cnico-profesional-en-Chile.pdf

Arrollo, C., y Pacheco, F. (2018). Los resultados de la Educación Técnica en Chile. Nota Técnica 3. Santiago de Chile: Comisión Nacional de Productividad. Recuperado de https://www.comisiondeproductividad.cl/wp-content/uploads/2018/06/NotaT\%C3\%A9cnica-3.-Educaci\%C3\%B3n.pdf

Carnoy, M. (1999). Globalización y reestructuración de la educación. Revista de Educación, (318), 145-162. Recuperado de http://www.educacionyfp.gob.es/dam/jcr:89f89292e282-4e35-9a5a-c774fdcf3e0c/re3180707707-pdf.pdf

CEDEFOP (2012). European Centre for the Development of Vocational Training Germany. VET in Europe: Country Report, 2012. Recuperado de 
MODELOS DE PROVISIÓN Y FORMACIÓN DUAL. UNA TAREA PENDIENTE PARA EL CASO DE LA ENSEÑANZA MEDIA TÉCNICO-PROFESIONAL EN CHILE / LOYOLA-BUSTOS

https://www.cedefop.europa.eu/en/publications-and-resources/country-reports/vetin-europe-country-reports

Consejo Asesor Presidencial para la Calidad de la Educación (2006). Informe Final. Santiago, Chile. Recuperado de: http://www.opech.cl/bibliografico/doc_movest/informe_final_consejo_asesor2.pdf

Dieter, E. (2013a). Germany's dual vocational training system: a model for other countries? Gutersloh, Alemania: Bertelsmann Stiftung. Recuperado de https://www.bertelsmannstiftung.de/fileadmin/files/BSt/Publikationen/GrauePublika tionen/GP_Germanys_dual_vocational_training_system.pdf

Dieter, E. (2013b). El sistema dual en Alemania. ¿Es posible transferir el modelo al extranjero? Barcelona, España: Fundación Bertelsmann. Recuperado de https://sanjose.diplo.de/blob/1520324/8dab2a985c4c9f7ab2a75ddfa35fa516/formacion-dualdata.pdf

Hargreaves, A., y Shirley, D. (2012). Las tres vías del cambio. En A. Hargreaves y D. Shirley. La cuarta vía. El prometedor futuro del cambio educativo. Barcelona: Octaedro. Pp. 25-49.

Hoeckel K. y Schwartz R. (2010). Learning for Jobs. OECD Reviews of Vocational Education and Training, Germany. París: OCDE. Recuperado de https://partners.aflcio.org/system/files/5_learning_for_jobs_oecd.pdf

Jacinto, C. (2013). Incluir a los jóvenes. Retos para la educación terciaria técnica en América Latina. Buenos Aires, Argentina: Instituto Internacional de Planeamiento de la Educación, UNESCO.

Larrañaga, O., Cabezas G. y Dussaillant, F. (2013). Informe completo de la educación técnico profesional. Santiago: PNUD.

Lauglo, J. (2006). Research for TVET Policy Development. Magdeburg, Alemania: UNEVOC - Inwent. 
REVISTA SABERES EDUCATIVOS, Nº 5, JULIO-DICIEMBRE 2020

López, M., Elizalde, L. y Rolando, R. (2015). Acceso a la educación superior de los estudiantes secundarios en Chile. SIES, (02) 1-22.

Messina G, Weinberg, P. e Irigoin, M. (1996). La educación técnica y la formación profesional. Santiago, Chile: UNESCO.

Mineduc (2007). Anuario Estadístico 2006-2007. Santiago: Mineduc.

Mineduc (2011). Educación Técnico Profesional en Chile: antecedentes y claves de diagnóstico. Santiago, Chile: Centro de Estudios, Ministerio de Educación. Recuperado de https://bibliotecadigital.mineduc.cl/bitstream/handle/20.500.12365/548/MONO466.pdf? sequence=1\&isAllowed=y

Mineduc (2013). Educación media técnico profesional: hallazgos del seguimiento de una generación. Serie Evidencia, 2(20).

Moodie, G. (2008). From vocational to higher education: An international perspective. Birmingham, Reino Unido: Open University Press.

Montecinos, M. J. (2017). Educación media técnico profesional: problematizando el discurso de la opción B. Cuaderno de Educación, (77), 1-6. Recuperado de http://mailing.uahurtado.cl/cuadernos_educacion_77/documentos/Actualidad_77\%2 0MJMontecinos\%20final.pdf

OCDE (2010a). Learning for Jobs. OECD Policy Review of Vocational Education and Training. París, Francia: OECD Publishing. Recuperado de http://www.oecd.org/education/innovation-education/learningforjobs.htm

OECD, (2010b) Preparándose para trabajar, el análisis de la OCDE sobre la educación y formación profesional- Alemania. Recuperado de https://www.oecdilibrary.org/education/preparandose-para-trabajar_9789264118478-es 
MODELOS DE PROVISIÓN Y FORMACIÓN DUAL. UNA TAREA PENDIENTE PARA EL CASO DE LA ENSEÑANZA MEDIA TÉCNICO-PROFESIONAL EN CHILE / LOYOLA-BUSTOS

Paredes, R., y Sevilla, M. P. (2015). Reforma de la Educación Superior Técnico-Profesional. En I. Sánchez (Ed.). Ideas en educación. Reflexiones y propuestas desde la UC. Santiago, Chile: Ediciones UC. Pp. 413- 444.

Rindfleish, E. y Meannig-Fortmann, F. (2015). Formación dual en Alemania. Formar técnicos por medio de la teoría y la práctica. Berlín, Alemania: Fundación Konrad Adenauer. Recuperado de https://www.kas.de/c/document_library/get_file?uuid=1535054a-e18a-ebf9-8204b883adda0115\&groupId=287914

Sepúlveda, L. (2008). Estado y perspectivas de la enseñanza media técnico profesional en Chile: un estudio sobre las orientaciones estratégicas predominantes de los actores. Proyecto FONIDE $N^{\circ}$ : F31083. Santiago, Chile: Ministerio de Educación. Recuperado de https://centroestudios.mineduc.cl/wpcontent/uploads/sites/100/2017/07/2008-CIDE-UAH-Sepulveda-3-3.pdf

Sepúlveda L. y Valdebenito, M. J. (2014a). ¿Las cosas claras? Aspiraciones de futuro y proyecto educativo laboral de jóvenes estudiantes secundarios. Estudios Pedagógicos, 40(1), 243-261.

Sepúlveda, L. y Valdebenito, M. J. (2014b). Aspiraciones y proyectos de futuro de estudiantes de enseñanza técnica- profesional ¿Es pertinente un sistema diferenciado en la educación media? Polis, (38), 597-620.

Sepúlveda, L., Sevilla, M. y Farías, M. (2014). ¿Y qué hay con la formación dual?: Alternativas para la educación media técnico profesional en Chile. Cuadernos de Educación, 58, 1- 3.

Sevilla M.P. (2017). Panorama de la Educación Técnica Profesional en América Latina y el Caribe. Serie Políticas Sociales 222. Santiago, Chile: CEPAL, Naciones Unidas. Recuperado de https://www.cepal.org/es/publicaciones/40920-panorama-laeducacion-tecnica-profesional-america-latina-caribe 
REVISTA SABERES EDUCATIVOS, Nº 5, JULIO-DICIEMBRE 2020

Sevilla, M. P., Farías, M. y Sepúlveda, L. (2013). Una nueva perspectiva para la educación técnica: condiciones y propuestas asociadas. Cuadernos de educación, (56), 1-4.

Sevilla, M. P., Farías, M. y Weintraub, M. (2014). Articulación de la educación técnico profesional: una contribución para su comprensión y consideración desde la política pública. Calidad en la Educación, (41), 83-117.

Urzúa, S. (2012). La rentabilidad de la educación superior en Chile ¿educación superior para todos? Documento de Trabajo 386. Santiago, Chile: Centro de Estudios Públicos. Recuperado de: https://www.cepchile.cl/cep/site/docs/20160304/20160304095900/rev125_SUrzua.p df

Van Breugel, G. (2014). Sistemas nacionales de formación profesional y capacitación. Una revisión de experiencias de países de la OCDE. Documentos de Proyecto. Santiago, Chile: CEPAL. Naciones Unidas. Recuperado de https://repositorio.cepal.org/handle/11362/36799

Villar, P. (2013). Educación media técnico profesional como una semilla de desarrollo. Estudios Nueva Economía III, 2(2) 35-42. Disponible en: https://estudiosnuevaeconomia.cl/team-view/estudios-nueva-economia-iii/ 